\title{
A Study on Cloud Simulation Tools
}

\author{
Kalpana Ettikyala \\ Assistant Professor \\ CSE Department \\ CBIT, Gandipet, Hyderabad, India
}

\author{
Y Rama Devi, Ph.D. \\ Professor and Head \\ CSE Department \\ CBIT, Gandipet, Hyderabad, India
}

\begin{abstract}
Cloud computing is an emerging technology, advancing quickly in IT due to the flexibility of using computing without buying any infrastructure but by using as pay-per-use model. It is risky to evaluate the performance of cloud environments as cloud computing adoption increases. The simulation technology has become a powerful tool in cloud computing research community to address these issues. Various cloud simulators have been precisely developed for testing and performance analysis of cloud computing environments. This paper presents a study, analysis and comparison of various existing cloud simulation tools.
\end{abstract}

\section{Keywords}

Cloud computing, Data centers, Cloud simulation tools.

\section{INTRODUCTION}

With the surging demands for computing resources and the quick development of virtual resource isolation technology [1], cloud computing has become a fascinating paradigm that can provision elastic services and on demand resources for various users based on pay-per-use model. Ease-of-use and close-to-practice simulators will be very helpful for in-depth research on cloud computing. Cloud simulators need many new requirements than traditional Grid [2,3] or Network simulators $[4,5]$.

The demand for resource isolation is high in cloud computing execution environment. For example, any cloud user execution environment will be in the form of virtual machine (VM) instances, each of which is allocated with various types of on-demand resources like CPU speed, memory sizes, etc.

The restrictions for cloud task execution are more than the grid task execution, which makes cloud simulation difficult. Generally the various types and amount of resource consumption by a cloud task may be associated to many factors like payment budgets, user bids and job priorities. For example, in Amazon EC2 resource allocation depends on user's bids and payment model.

Cloud application's execution may not show regular rules in cloud computing environment which is different from HPC (High Performance Computing) and grid applications. Grid/HPC applications are mostly computation intensive which requires heavy workload on computation than other type resources. For example Map Reduce application in cloud environment is $\mathrm{I} / \mathrm{O}$ intensive distributed program. Google trace display that many google applications demand memory size than the CPU rate.

With the cloud simulation tools performance analysts can analyze the system behavior by concentrating on specific component's quality under different scenarios [6].

This paper presents a study, analysis and comparison of various existing cloud simulation tools. In the next section, fifteen different cloud simulators are analyzed and discussed.
Finally they are compared with one another in different characteristics like programming language supported, platform supported, network limitation, type of the simulator, etc.

\section{CLOUD SIMULATION TOOLS 2.1 CloudSim}

This simulator is an extensible toolkit which enables the simulation, modeling and experimentation of cloud system its infrastructures and application environments of single and internetworked clouds [7]. It is a feasible solution if access to hardware is finite. This simulation software supports modeling of cloud data centers with different hardware configurations, helps in modeling user applications with independent tasks, supports design and analysis of various VM (virtual machine) provisioning, scheduling policies. It also enables to model power consumption, network behavior.

CloudSim implemented application provisioning techniques can be extended easily with less efforts. This software helps researchers to concentrate on particular design issues without considering the low level details of cloud infrastructures and services [8]. It introduces preconfigured machines which are designed to execute common open source robotic tools. SimJava , a toolkit based on discrete event simulation kernel which is present at lowest level of cloudsim allows to develop models of complex systems. It also provides facilities to represent the simulated objects as animated icons.

\subsection{CloudAnalyst}

This tool is derived from cloudsim by extending some of its features [9]. Unlike cloudsim, cloudanalyst is graphical in nature which has better visualization results. It also separates the simulation experimentation from programming exercise. It also enables perform simulations and to manage a series of simulation experiments with slight parameters variations in a fast and easy manner. It can also examine the behavior of large scaled internet application in a cloud environment.

\subsection{GreenCloud}

It is a packet level cloud network simulator which concentrates on cloud communication [10]. It is the extension of network simulator (NS2). This simulation tool collects fine grained information about energy consumed by various communication and computing resources (like servers, links, switches, etc.) of a data center in unique fashion. It also allows the examination of workload distributions. It is the application of designing, manufacturing and usage of computing resources with minimum environmental harm. This tool can reduce power consumption by consolidating workloads with data center virtualization. Complete TCP/IP protocol is implemented by this simulator which makes different communication protocols to be integrated with simulation. But, GreenCloud simulator takes high simulation time and it requires more memory, which makes its scalability to be limited to small data centers. 


\subsection{NetworkCloudSim}

This simulation tool is the extension of cloudsim. It supports high performance computing applications [11]. CloudSim network layer is implemented by Network Topology class and this class is used by NetworkCloudSim tool which reads a BRITE file and produces a topological network. Topology file comprises of nodes and number of entities in simulation, which enables users to raise the scale of simulation without any modification of topology file. And each BRITE node should be mapped to one entity at a time which enables proper working of network simulation. This tool allows to model cloud data centers with fast and scalable simulations by sharing bandwidth.

\subsection{EMUSim}

It is an unified architecture [12] which predicts cloud service's behavior to a high standard. This tool is built on Automated Emulation Framework (AEF) for emulation and on CloudSim for simulation. It automatically collects application behavior information via emulation and then uses this data to produce the particular simulation model. This generated simulation model will be used to construct a scenario which will be closer to original target production environment of application request patterns and computing resources. VM (virtual machine) related information like VM location, number of VMs per host, etc. in a given time is not required by EMUSim.

\subsection{MDCSim}

This tool can simulate hardware characteristics of various components available in a data center and helps to estimate the power consumption. The data center topology is represented as directed graph [13] by the network package of MDCSim and this tool is very prominent because of less simulation overhead.

\subsection{GDCSim}

Green Data Center Simulator [14] combines the modular and extensible entities. This tool helps to study the energy efficiency of data centers beneath various data center geometries, platform energy management schemes, scheduling algorithms and workload characteristics. It helps to design green data center by capturing the interdependencies between online resource management and physical behavior of data centers

\subsection{SPECI}

Simulation Program for Elastic Cloud Infrastructures (SPECI) helps to analyze different scalability and performance features of data centers [15]. Data centers expand in nonlinear fashion which makes a need to analyze the behavior and SPECI helps here to analyze behavior of such data centers. This tool can be used to monitor the inconsistencies that rise when failures occur in data centers. SPECI helps to manage distributed data center when data center size and failure rate increases.

\subsection{CDOSim}

Cloud Deployment Option (CDO) simulator, it can simulate SLA violations, costs and response times of a cloud deployment. It is a decision maker about cloud provider, runtime adaption schemes, components deployment (it includes formation of new components from existing components) and instances configuration of VM. It can simulate cloud deployments which were reverse engineered to KDM models. This tool allows the combination of fine grained models and it helps to represent the user's perspective rather than cloud provider perspective. It can tradeoff between costs and performance [16] or it can be used to compare runtime reconfiguration plans.

\subsection{TeachCloud}

This tool is meant for education purpose where one can alter cloud configuration and can perform simple experiments with the help of its graphical interface [17]. It uses cloudsim as design platform with some enhancements on the top of cloudsim like GUI, cloud workload generator, cloud network models(such as VL2, Portland, Dcell, etc.), new models related to SLA and Business Process Management(BPM).

\subsection{1 iCanCloud}

This simulator is based on SIMCAN. It is a framework for huge storage networks. For an application in a specific hardware it can tradeoff between costs and performance to give the idea for the user about the costs involved. It concentrates on policies which charge users as pay-per-use model [18]. This tool has full GUI which facilitates easy design and execution of experiments. It also supports experiment execution in parallel over several machines.

\subsection{GroudSim}

GroudSim [19] is an event based simulator which requires a simulation thread for grid and cloud environment scientific applications based on a scalable simulation independent discrete event core. It focuses on IaaS and it is easy extendable to support other cloud level services like Paas, Daas. Groudsim can be combined with ASKALON environment to simulate experiments in the same environment which is used for real applications. It provides set of features to handle complex simulation scenarios like background load on resources, cost calculation, task execution on leased computing resources.

\subsection{DCSim}

This tool develops data center management techniques. It concentrates on virtualized data centers. It will focus on transactional and continuous workloads. This simulator can model dependencies between VMs which belongs to multitiered application [22]. It can also simulate replicated VMs sharing incoming workload. It can easily measure the SLA achievement.

\subsection{FlexCloud}

This tool (flexible and scalable simulator) can simulate resource scheduling and performance evaluation of VM allocation in cloud data centers [20]. It can evaluate various scheduling policies and algorithms, can simulate cloud data centers initialization, can model VM allocation requests and performance evaluation of different scheduling algorithms. It concentrates on IaaS, has user friendly interfaces for replaying and customized configurations and it can model VM migrations. This simulation software supports large scale simulations by reducing the computing time and memory consumption. It has unified features to support public cloud providers, energy efficient scheduling and load balancing.

\subsection{GloudSim}

Google Trace based Cloud Simulator with Virtual Machines [21], it is a distributed cloud simulator which is based on virtual machines. It can emulate the tasks that consume resources like CPU rate, memory, etc. which dynamically changes, with the closer real values. This tool can emulate different events like kill/evict, etc. accurately based on trace. 
To support various research demands, it can also be able to emulate more useful and complex cases beyond the actual trace.

\section{COMPARISONS OF MAJOR CLOUD SIMULATION TOOLS}

Table 1: Comparison of Cloud Simulation Tools

\begin{tabular}{|c|c|c|c|c|c|}
\hline Simulator & Platform & $\begin{array}{c}\text { Programming } \\
\text { language }\end{array}$ & Networking & Simulator type & Availability \\
\hline CloudSim & GridSim & Java & Limited & - & Open source \\
\hline CloudAnalyst & CloudSim & Java & Limited & Event based & Open source \\
\hline GreenCloud & NS2 & $\mathrm{C}++/ \mathrm{OTCL}$ & Full & Packet level & Open source \\
\hline NetworkCloudSim & CloudSim & Java & Full & Packet level & Open source \\
\hline EMUSim & AEF,CloudSim & Java & Limited & Event based & Open source \\
\hline MDCSim & CSIM & $\mathrm{C}++/$ Java & Limited & Event based & Commercial \\
\hline CDOSim & - & Java & - & - & - \\
\hline TeachCloud & - & Java & - & - & - \\
\hline SPECI & SimKit & Java & - & - & - \\
\hline DCSim & - & Java & - & - & - \\
\hline GroudSim & - & Java & - & - & - \\
\hline iCanCloud & OMNET,MPI & $\mathrm{C}++$ & - & - & Open source \\
\hline FlexCloud & Any & Java & - & - & Open source \\
\hline
\end{tabular}

\section{CONCLUSION}

Cloud testing is one of the happening research topic in cloud computing. Simulation based approach has become well liked technology to evaluate cloud computing systems, their security and performance and application behaviors. Several simulators have been particularly developed for evaluation and performance analysis of cloud environments. This paper has presented a study and comparison of different simulators used in cloud computing. Each simulator has its own characteristics which makes it different from others.

\section{REFERENCES}

[1] D. Gupta, L. Cherkasova, R. Gardner, and A. Vahdat, "Enforcing performance isolation across virtual machines in XEN," in Proceedings of the 7th ACM/IFIP/USENIX Int'l Conf. On Middleware (Middleware'06), 2006, pp. 342-362.

[2] R. Buyya and M. Murshed, "GridSim: A toolkit for the modeling and simulation of distributed resource management and scheduling for Grid computing," Journal of Concurrency and Computation: Practice and Experience (CCPE), vol. 14, no. 13, pp. 1175-1220, 2002.

[3] H. Casanova, A. Legrand, and M. Quinson, "SimGrid: A generic framework for rarge-scale distributed experiments," in Proceedings of the 10th International
Conference on Computer Modeling and Simulation (UKSIM'08), 2008, pp. 126-131.

[4] Alberto Montresor and Mark Jelasity., "PeerSim: A scalable P2P simulator," in Proceedings of the 9th Int. Conference on Peer-to-Peer (P2P'09), pp. 99-100, Seattle, Sept., 2009.

[5] S. Mccanne, S. Floyd, and K. Fall, "The Network Simulator NS-2", http://www.isi.edu/nsnam/ns/.

[6] Xiaoying Bai, Muyang Li, Bin Chen, W.T. Tsai \& J. Gao (2011), "Cloud Testing Tools", Proceedings of the 6th IEEE International Symposium on Service Oriented System Engineering, Pp. 1-12.

[7] R.N. Calheiros, Rajiv Ranjan, Anton Beloglazov, Cesar A.F. De Rose \& Rajkumar Buyya (2011), "CloudSim: A Toolkit for Modeling and Simulation of Cloud Computing Environments and Evaluation of Resource Provisioning Algorithms", Software: Practice and Experience, Vol. 41, No. 1, Pp. 23-50.

[8] B. Wickremasinghe, "CloudAnalyst: a CloudSim-based tool for modeling and analysis of large scale cloudcomputing environments," MEDC Project Report, 2009.

[9] B. Wickremasinghe (2009), "CloudAnalyst: A CloudSim based Tool for Modeling and Analysis of Large Scale 
Cloud Computing Environments", MEDC Project Report.

[10] D. Kliazovich, P. Bouvry \& S.U. Khan (2010), "GreenCloud: A Packet-Level Simulator of Energy Aware Cloud Computing Data Centers", IEEE Global Telecommunications Conference,Pp.1-5.

[11] R. Buyya, R. Ranjan \& R.N. Calheiros (2009), "Modeling and Simulation of Scalable Cloud Computing Environments and the CloudSim Toolkit: Challenges and Opportunities", The International Conference on High Performance Computing and Simulation, Pp. 1-11.

[12] R.N. Calheiros, Marco A.S. Netto, César A.F. De Rose \& Rajkumar Buyya (2012), "EMUSIM: An Integrated Emulation and Simulation Environment for Modeling, Evaluation, and Validation of Performance of Cloud Computing Applications", Software: Practice and Experience, Pp. 1-18.

[13] Dr. Pawan Kumar \& Gaganjot Kaur , "Study of Comparison of Various Cloud Computing Simulators", IITT College of Engineering \& Technology, 2nd National Conference in Intelligent Computing \& Communication, GCET Greater Noida, India

[14] Gupta Sandeep K.S. and Gilbert Rose Robin, "GDCSim An Integrated Tool Chain for Analyzing Green Data Center Physical Design and Resource Management Techniques", 2011 IEEE, pp.1-8.

[15] Sriran Ilango,"SPECI, a simulation tool exploring cloudscale data centres"

[16] F. Fittkau, S. Frey, W. Hasselbring, "Cloud user-centric enhancements of the simulator cloudsim to improve cloud deployment option analysis", Proceedings of the 1st European conference on Service-Oriented and Cloud Computing, ESOCC'12, 2012.

[17] Y. Jararweh, Z. Alshara, M. Jarrah, M. Kharbutli, M. Alsaleh, "Teachcloud: a cloud computing educational toolkit", Proceedings of the 1st International IBM Cloud Academy Conference (ICA CON 2012), IBM, Research Triangle Park, NC, USA, 2012.

[18] A. Nunez, J.L. Vazquez-Poletti, A.C. Caminero, G.G. Castane, J. Carretero, I.M. Llorente, "iCanCloud: a flexible and scalable cloud infrastructure simulator", Journal of Grid Computing 10:1, 2012, page:185-209.

[19] Simon Ostermann, Kassian Plankensteiner, Radu Prodan, and Thomas Fahringer, "GroudSim: An Event Based Simulation Framework for Computational Grids and Clouds", M.R. Guarracino et al. (Eds.): Euro-Par 2010 Workshops, pp. 305-313, 2011. Springer- Verlag Berlin Heidelberg, 2011

[20] FlexCloud: A Flexible and Extendible Simulator for Performance Evaluation of Virtual Machine Allocation Minxian Xu, Wenhong Tian, Xinyang Wang, Qin Xiong Dr. Tian, Mr. Wang and Miss Xiong are in the school of Computer Science, University of Electronic Science and Technology of China (UESTC), 610054; Mr. Xu is in the school of software engineering of UESTC.

[21] GloudSim: Google Trace based Cloud Simulator with Virtual Machines by Sheng Di, Franck Cappello 1INRIA, Argonne National Laboratory, USA

[22] DCSim: "A Data Centre Simulation Tool"- Gaston Keller, Michael Tighe, Hanan Lutfiyya and Michael Bauer -' 2013 IFIP/IEEE International Symposium on Integrated Network Management (IM2013). 